\title{
QUANTILE REGRESSIVE FISH SWARM OPTIMIZED DEEP CONVOLUTIONAL NEURAL LEARNING FOR RELIABLE DATA TRANSMISSION IN IOV
}

\author{
S. Suguna Devi and Dr. A. Bhuvaneswari \\ Department of Computer Science, Cauvery College For Women, Trichy-18, India \\ Affiliated to Bharathidasan University
}

\begin{abstract}
Route path identification on the Internet of Vehicles (IoV) is complicated due to the nature of high dynamic mobility, bandwidth constraints, and traffic load. A vehicle present on the IoV communicates with each other to find the status of the road and location of other vehicles for reliable data transmission. However, the existing routing algorithm does not effectively improve the packet delivery ratio and reduce the delay. To resolve these issues, A Quantile Regressive Fish Swarm Optimized Deep Convolutional Neural Learning (QRFSODCNL) technique is introduced reliable data transmission with minimum end to end delay in IoV. The Deep Convolutional Neural Learning uses multiple layers such as one input layer, three hidden layers, and one output layer for vehicle location identification and optimal route path discovery. The different node characteristics of vehicle nodes are analyzed in the hidden layers using the quantile regression function. Depends on the regression analysis, the neighbouring node is identified with minimal time. To improve the throughput and reduce the packet loss rate, the artificial fish swarm optimization technique is applied to choose the best route among the population based on the fitness function. Simulation is carried out to analyze the performance of QRFSODCNL technique and existing methods with different metrics such as packet delivery ratio, packet loss rate, average end to end delay, and throughput. The discussed outcome proves that the QRFSODCNL technique outperforms well as compared to the stateof-the-art methods.
\end{abstract}

\section{KEYWORDS}

IoV, Deep Convolutional Neural Learning, neighbouring location identification, Quantile Regression, multicriteria artificial fish swarm optimization, optimal route path identification

\section{INTRODUCTION}

With the development of a transportation system, I have emerged to facilitate more convenient and fast services. I adopt a communication network to realize the intercommunication and coordination between vehicles. It is designed to cater to the needs of the automotive industry and has become a popular and crucial platform with information interaction among vehicles, pedestrians, drivers, and city infrastructure. IoV permits vehicles to exchange information with infrastructures using Vehicular Ad Hoc Networks (VANETs). This generates a network with intelligent devices as participants. It observes driving habits and gives recommendations, notifications of problems, or emergency alerts to the driver. However, there is a need for improving the communication speed between the vehicles. The optimal route path identification is incorporated to avoid route path failure which plays a major role in IoV. Deep learning is implemented to improve vehicle communication with minimum loss and delay.

The community aware mechanism [1] on the Internet of vehicles (CVs) was developed to perform the data communication between the vehicles. Though the mechanism minimizes the 
delay, the higher packet delivery ratio was not achieved. The quality of service-aware routing algorithm (QRA) [2] is proposed to identify the optimal path. However, the designed QRA algorithm failed to perform reliable data communication.

To distribute data packets based on bandwidth constraints, an Energy Efficient Multicast routing protocol based on Software Defined Networks and Fog computing for Vehicular networks (EEMSFV) was proposed [3]. The method failed to improve network throughput.

An Identical Destination Based C ommunity [4] on the Internet of Vehicles (IDCIoV) was developed to find the optimal route path. The method failed to reduce the end-to-end delay while performing the data communication. A distributed replication-based data dissemination algorithm [5] was proposed for efficient data transmission between the vehicles. But the performance of the packet loss rate was not reduced. A metaheuristic dragonfly-based clustering algorithm was introduced [6] for cluster-based packet route optimization to improve the transmission. But the performance of the algorithm was not improved for mobility awareness.

A grey wolf optimization-based clustering algorithm [7] was developed for reliable data transmission. The designed algorithm failed to consider the bandwidth constraints for efficient data transmission. The Ant colony optimization (ACO) and Particle swarm optimization (PSO) Algorithms were introduced in [8] for increasing the data communication between the vehicles. But it failed to analyze the node characteristics for neighbouring node identification. An enhanced intelligent hybrid routing protocol using improved fuzzy and cuckoo algorithms was developed [9] to discover the stable path between a source and destination. But effective neighbouring node location identification remained unaddressed. A Software-Defined Cognitive Network for IoV (SDCIV) was introduced [10] for efficient data communication. The designed method did not use any optimization method for optimal route path identification.

The objective of the proposed QRFSODCNL technique is summarized as follows,

To improve reliable data packet delivery in IoV, the QRFSODCNL technique is introduced by identifying the neighbouring location and optimal path discovery using different layers. In the hidden layers, the different characteristics of the vehicle nodes such as distance, signal strength, direction, and energy are analyzed using the quantile regression function. Based on the regression analysis, the neighbouring nodes are identified. Then the multiple route paths between the source and destination are constructed for reliable data transmission. This helps to minimize the end-to-end delay.

To improve the network throughput and minimize the packet loss, an artificial fish swarm optimization technique is introduced with multicriteria such as path length and bandwidth availability. The optimization technique selects the best route and obtains the results at the output layer for data transmission among the population based on the fitness function.

The structure of the article is ordered as follows: Section 2 reviews the related works. Section 3 describes the proposed methodology. The simulation settings are provided in section 4 . The results and discussion are provided in section 5. Section 6 concludes the paper.

\section{RELATED WORK}

A bio-inspired unicast routing protocol was introduced in [11] to select the next hops for data transmission between the vehicles. Though the designed protocol reduces delay, the delivery ratio was not improved. A micro artificial bee colony (MABC) algorithm was designed [12] to 
enhance the network lifetime and reduce delay cost. The designed algorithm failed to analyze the various metrics such as packet delivery and loss rate.

A Path Transmission Costs-based Multi-lane Connectivity Routing protocol (PTCCR) was developed [13] for finding the neighbour location and optimal path. However, it failed to use the swarm intelligence for packet transmission with minimum delay. A statistical framework method was developed [14] for optimizing the routing parameters to improve vehicle communication. But the designed model did not perform the relay node selection to evaluate the quality of the link connection.

A hybrid relay node selection technique was developed [15] for message distribution with minimum delay. However, the multiple characteristics of the relay nodes were not considered. The clustering and position-based broadcast approaches were introduced in [16] for data transmission with lesser delay. But the network throughput was not improved. A reliable emergency message dissemination protocol was proposed [17] with less end to end delay. But the packet delivery ratio was not improved.

A coalitional game theory-based clustering algorithm was designed [18] to improve the data packet communication. Though the algorithm minimizes the delay, the packet loss rate was not minimized. An ant colony optimization was developed [19] to improve the accuracy of path identification and also minimize the packet loss. The energy-aware path identification remained unaddressed.

A Heterogeneous IoV Architecture was introduced [20] for transmitting the data packets. The neighbouring node identification was not performed. A hybrid emergency message transmission (HEMT) method was developed [21] for improving the transmission efficiency. The method failed to consider energy-aware message transmission.

A road aware approach was designed [22] to forward packets among source and destination. In the proposed approach, roads are partitioned into segments depends on an intersection with unique ids. Vehicles share their information via the hello beacons to discover the best path among source and destination. But, the delay was more. In [23], two member-centric protocols were introduced to ensure the reliability of data forwarding for multiple-source to single-destination. A novel Heterogeneous architecture was presented in [24] for data transmission from vehicle to infrastructure (V2I).

\section{Proposed System}

The Quantile Regressive Fish Swarm Optimized Deep Convolutional Neural Learning (QRFSODCNL) is introduced for improving the reliable data transmission in IoV with minimum delay. Probit Regressive Chaotic Bio-inspired Grey Wolf Optimization (PRCBGWO) technique enhances data transmission although the energy-aware neighbour location identification techniques are not used to decrease the packet loss and delay. Hence, the QRFSODCNL technique is proposed for identifying the neighbouring location by considering the energy parameters. In the proposed QRFSODCNL technique, deep convolutional neural learning is used to achieve efficient data transmission by selecting an optimal route path based on the fitness measurement. Deep convolutional neural learning (DCNL) is a machine learning algorithm, it includes multiple layers to extract features from input data, and better results are attained in deeper architectures where each layer is pre-trained with an unsupervised learning algorithm. It is a type of machine learning algorithm that utilizes the quantile regression algorithm for neighbouring node identification. DCNL assigns importance to each data in input and can differentiate one from the other. DCNL was inspired by the Neurons in the Human Brain. The 
role of DCNL is to reduce the vehicle data which is easier to process, without losing features to attain good prediction. At first, DCNL creates the number of neurons and assigns random numerical values (i.e., Weights). If the network did not accurately analyze the node characteristics, an algorithm would adjust the weights.

The IoV enabled vehicle nodes are organized in an undirected graph $g\left(V_{t}, L_{n}\right)$ where the vehicle nodes $v_{1}, v_{2}, v_{3}, \ldots . v_{n}$ are represented by $V_{t}$ which are distributed over a squared area of ' $m *$ $m^{\prime}$ ' and ' $L_{n}$ ' denotes a connection between the pair of nodes. To initiate the data transmission, the source node $(\mathrm{SV})$ finds one-hop neighbours $N n_{1}, N n_{2}, N n_{3}, \ldots . N n_{n}$ towards the destination node $(D V)$.After that, multiple route pathsp $\mathrm{p}_{1}, \mathrm{p}_{2}, \mathrm{p}_{3}, \ldots ., \mathrm{p}_{n}$ are established between the $S V$ and $D V$. Subsequently, an optimal path $\left(\mathrm{P}_{\mathrm{th}}\right)$ among the multiple paths is identified to improve the data transmission in IoV. With the help of the above system model, the proposed QRFSODCNL technique is designed.

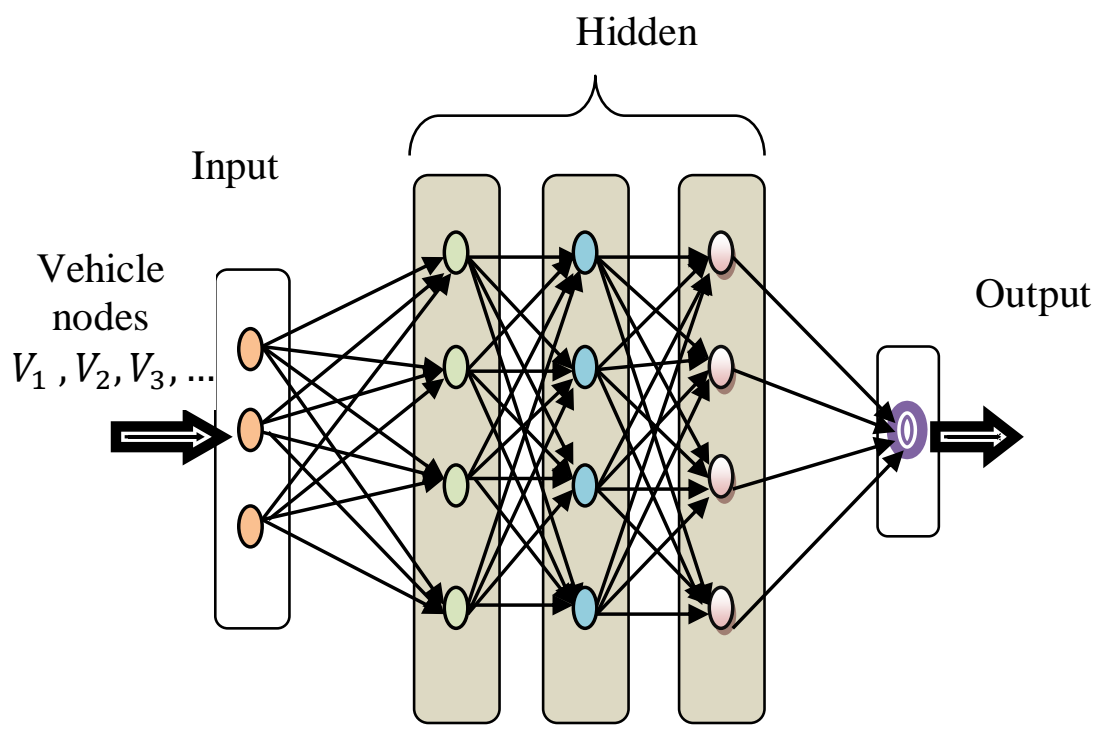

Figure 1. Structure of Deep Convolutional Neural Learning

Figure 1 illustrates the structure of the deep convolutional neural learning with one input layer, three hidden layers, and one output layer. The vehicle nodes are given to the input layer $v_{1}, v_{2}, v_{3}, \ldots . v_{n}$ at the time. Deep neural learning comprises the neurons-like nodes that are fully connected to the deep convolution layers with the adjustable weights used to learn the features. Then, the inputs are transferred into the first hidden layer. In that layer, the node characteristics are analyzed to find the neighbour location for data transmission. Let us consider the number of vehicle nodes $v_{1}, v_{2}, v_{3}, \ldots . v_{n}$ and defines the source vehicle $(S V)$ and destination vehicle $(D V)$.

\subsection{Neighbouring node location identification}

The neighbouring node location identification is performed using different characteristics such as distance, signal strength, angle of direction, and energy. 
- Distance

The distance is measured based on the time of arrival which is the time difference between the beacon message transmission and beacon message reception between the source vehicle and the other vehicles in the network.

$$
d=\left(\mathrm{T}_{\mathrm{B}}-\mathrm{T}_{\mathrm{r}}\right)(1)
$$

Where $d$ represents a distance between the vehicle nodes, $\mathrm{T}_{\mathrm{B}}$ represents a time for beacon message transmitted from source to other nodes, and $\mathrm{T}_{\mathrm{r}}$ denotes a reply message arrival time from the other nodes.

\section{- Received signal strength}

After finding the distance, the received signal strength of the mobile node is calculated as follows,

$$
R_{S S}=10 * \log _{10}\left(\frac{P_{T}}{P_{R}}\right)
$$

Where $R_{S S}$ represents a received signal power of the vehicle node, $P_{T}$ represents a measured power, $P_{R}$ denotes a reference power.

\section{- Angle of direction}

The angle of direction is used to find the direction of the movable vehicle nodes from the source node. Let us consider, the coordinate of the source and the other vehicle nodes are $\left(\mathrm{u}_{1}, \mathrm{v}_{1}\right)$ and $\left(\mathrm{u}_{2}, \mathrm{v}_{2}\right)$. The angle of direction is computed using the following mathematical equations,

$$
\alpha=\tan ^{-1}\left(\frac{u_{2}-u_{1}}{v_{2}-v_{1}}\right)
$$

Where $\alpha$ represents the angle between the two vehicles.

\section{- Energy}

Energy is the major characteristics of the vehicle nodes to improve the transmission. Therefore, the energy of the vehicle node is measured as the product of the power and the time.

$$
E=P_{\text {power }} * T_{\text {time }}
$$

Where $E$ denotes energy, $P_{\text {power }}$ represents the power, $T_{\text {time }}$ denotes a time. The residual energy of the vehicle node is calculated as a difference between the total energy of the vehicle node and the consumed energy.

$$
E_{\text {res }}=E_{t}-E_{c d}
$$

Where $E_{r e s}$ represents the residual energy, $E_{t}$ denotes total energy, $E_{c d}$ is the consumed energy. With the above-said characteristics

$$
W=d_{i}<d_{j} \& \& R_{s s i}<R_{s s j} \& \& \text { Samedirection } \& \& E_{\text {res }}>E_{t h}(6)
$$

Where denotes a function which identifies the neighbouring location based on the comparison. The quantile regression is a statistical technique to analyze the node characteristics as follows. 


$$
y=Q\left(V_{1}, V_{2}, V_{3}, \ldots . V_{n} \mid W\right)=x_{t} * W
$$

Where $y$ denotes a regression output, $Q$ denotes a condition of the Quantile of the dependent variable $(\mathrm{y}), x_{t}$ denotes independent variables (i.e. Nodes), $W$ denotes a vector of parameters (i.e. $W$ ). The Quantile regression analyses the nodes with their characteristics and returns the values $(0,1)$. If the result is higher than the threshold, then the node is chosen as the neighbouring node. Subsequently, all the neighbouring locations are identified from source to destination vehicles. After finding the neighbouring nodes, the route paths from source to destination are established at the second hidden layer by distributing the two control messages namely request (req) and reply $(r e p)$. The source node distributes a request to the selected neighbouring nodes and the destination vehicle node. After receiving the request messages, the node sends reply messages back to the source node. In this way, the multiple route paths from source to destination are created.

\subsection{Optimal route path identification}

The output of the second hidden layer is transferred as input into the third hidden layer to find the best optimal route path for reliable data transmission. The optimal route path identification is performed using Multi-criteria Artificial Fish Swarm Optimization. The Fish Swarm Optimization is a swarm intelligence that worked based on the population and stochastic search. Swarm Intelligence is defined as the collective behavior of living mammals like birds, fishes, ants, and so on. The behavior of the animal is to find their food source (i.e. prey). On the contrary to the existing optimization algorithm, the proposed optimization algorithm is to provide the best approach which has a higher convergence speed, flexibility, and higher accuracy. Artificial fish swarm is a metaheuristic technique that works based on different behaviours like prey, swarm, and follows. Here the Artificial fish swarm is related to the multiple paths $\mathrm{p}_{1}, \mathrm{p}_{2}, \mathrm{p}_{3}, \ldots . \mathrm{p}_{\mathrm{n}}$ and multi-criteria referred to the multiple objective functions such as path length i.e. distances, bandwidth availability, node buffer capacity. Based on the multi-criteria, an optimal route path (i.e. artificial fish) is selected among the population.

By applying the meta-heuristic artificial fish swarm optimization, the population of ' $n$ ' artificial fish swarms (i.e. available paths) is randomly distributed in the search space.

$$
V=\mathrm{p}_{1}, \mathrm{p}_{2}, \mathrm{p}_{3}, \ldots . \mathrm{p}_{\mathrm{n}}
$$

After the initialization, the fitness is calculated for each fish swarm in the current population. The fitness is calculated based on multiple objective functions such as path length, bandwidth availability, and node buffer capacity.

The path length is measured based on distance from the source to the destination. Let us consider the coordinate of the two source vehicle and destination vehicles are $\left(\mathrm{x}_{1}, \mathrm{y}_{1}\right)$ and $\left(\mathrm{x}_{2}, \mathrm{y}_{2}\right)$ respectively. The distance between two points is estimated as

$$
D=\sqrt{\left(x_{2}-x_{1}\right)^{2}-\left(y_{2}-y_{1}\right)^{2}}
$$

Where $D$ is the distance between the source vehicle and destination vehicle?. Then the available bandwidth is calculated as follows,

$$
b w_{a}=\left(b w_{t t}-b w_{u}\right)
$$

Where, $b w_{a}$ denotes an available bandwidth of the route path from source vehicle to destination vehicle, $b w_{t t}$ denotes a total bandwidth, $b w_{u}$ denotes a utilized bandwidth. 
The node buffer capacity is computed for determining the node status. To calculate network load, the maximum buffer queue length is taken to lessen packet drops due to collision in the receiver node.

$$
B_{i}(t)=\frac{B_{\max }-B_{n}(t)}{B_{\max }}
$$

From (11), $B_{i}(t)$ is an average buffer capacity, $B_{\max }$ is a maximum buffer size, $B_{n}(t)$ denotes the number of packets in buffer queue at the time. If the buffer queue length is higher than the threshold, the node buffer size is full. In this case, the other alternative the node is chosen to forward the data packet depends on the fitness function. If the node buffer size is empty, it lessens routing to load that results in the master node lessen network traffic and enhances packet delivery. Then the fitness is measured based on these three objective functions.

$$
F(x)=\left\{(\min D) \& \&\left(b w_{a}>\delta\right) \& \&\left(B_{i}(t)<B_{t}\right)\right\}
$$

Where $F(x)$ denotes a fitness function, $\delta$ denotes a threshold for the bandwidth availability. $B_{t}$ refers to the threshold for buffer capacity. Based on the fitness value, three different behaviours of the fish such as search or prey, swarm, and follow are analyzed to identify the global best solution.

\section{Search or prey behaviour}

Prey is a fundamental behavior of the artificial fish that is used to find the food source (i.e multiple objective functions) in the search space. Generally, the fish identify the food source in the water is based on their vision or sense. Let us consider the current position of the fish is $F_{i}$ and the updated position is $F_{i}(t+1)$. If the fitness of one fish is higher than the other i.e. $F\left(x_{a}\right)<F\left(x_{b}\right)$, then the search or prey behavior is executed and then the position updates are expressed as follows

$$
F_{i}(t+1)=F_{i}(t)+R * S *\left(\frac{\left(F_{b}-F_{a}\right)}{\left\|F_{b}-F_{a}\right\|}\right)
$$

Where $F_{i}(t+1)$ represents an updated position of artificial fish, $F_{i}$ represent the current position of the artificial fish, $R$ is a random number $(0<R<1), S$ that represents a moving step of the artificial fish that is a random positive number, $\left\|F_{b}-F_{a}\right\|$ is the visual distance between the position of the ' $b$ ' fish and the position of the ' $a$ ' fish.

\section{Swarm behaviour}

In swarm behavior, the artificial fishes are moved in the form of a group for avoiding the risks. Let us consider the current position of the artificial fish is $F_{i}$, the center position of the several fish is represented by $F_{c}$. The swarm behavior of the fish is executed.

$$
F\left(x_{c}\right)<F\left(x_{a}\right) \& \&\left(\frac{N_{b}}{N}<\tau\right)
$$

Where $F\left(x_{c}\right)$ is the fitness of artificial fish at the center position, $N_{b}$ denotes the number of companions within the current neighbourhood, $N$ denotes a total number of artificial fishes, $\tau$ denotes a crowd factor $(0<\tau<1)$. Therefore, the position of artificial fish is updated as follows,

$$
F_{i}(t+1)=F_{i}(t)+R * S *\left(\frac{\left(F_{c}-F_{a}\right)}{\left\|F_{c}-F_{a}\right\|}\right)(15)
$$


Where $F_{i}(t+1)$ represents an updated position of artificial fish, $F_{i}(t)$ denotes a current position, $R$ represents a random number $(0<R<1)$, Sdenotes a movement steps of the fish which is a random positive number, $\left\|F_{c}-F_{a}\right\|$ is the visual distance between the central position of the fish and the current position of the fish.

\section{Follow behaviour}

Due to the movement of the fish swarm, when a single fish or group of fishes discovers their food, the neighbourhood trails and reaches the food in a fast manner. Let $F_{i}$ be the current position, and it uses the companion $\mathrm{F}_{\mathrm{j}}$ in the neighbourhood. If $\left(x_{b}\right)>F\left(x_{a}\right) \& \&\left(\frac{N_{b}}{N}<\tau\right)$, then the following behavior of the artificial fish is executed which indicates that the companion $x_{b}$ the state has maximum food concentration (i.e. higher fitness value). The position update is given below,

$$
F_{i}(t+1)=F_{i}(t)+R * S *\left(\frac{\left(F_{\max }-F_{a}\right)}{\left\|F_{\max }-F_{a}\right\|}\right)(16)
$$

Where $F_{i}(t+1)$ represents an updated position of artificial fish, $F_{i}(t)$ is the current position, $F_{\max }$ denotes a position having the best fitness inside the visual, $R$ is a random number $(0<R<$ $1)$, Srepresents a moving step of the artificial fish which is a random positive number, $\left\|F_{\max }-F_{a}\right\|$ is the visual distance between the central position of the fish having the maximum fitness function and the position of the ' $a$ ' the fish. Finally, the old artificial fish is replaced with a new optimal one based on their fitness. This process is iterated until it reaches the maximum iteration. The hidden layer output is indicated as ' $H(t)$ '.

$$
H(t)=\omega_{1} * x_{t}+\omega_{h} * H(t-1)
$$

Where $H(t)$ represents an output of a hidden layer. $H(t-1)$ ' indicates output from hidden layer 2 and ' $\omega_{h}$ ' is a weight of the hidden layer, $\omega_{1}$ is a weight among input and the hidden layers are the input. In equation (16), the symbol ' $*$ ' denotes a convolution operator. Finally, the optimal route path is obtained at the output layer. Then the source node transmits the data packets along the path to improve the packet delivery and minimizes the packet loss. The algorithmic process of deep learning is described as follows,

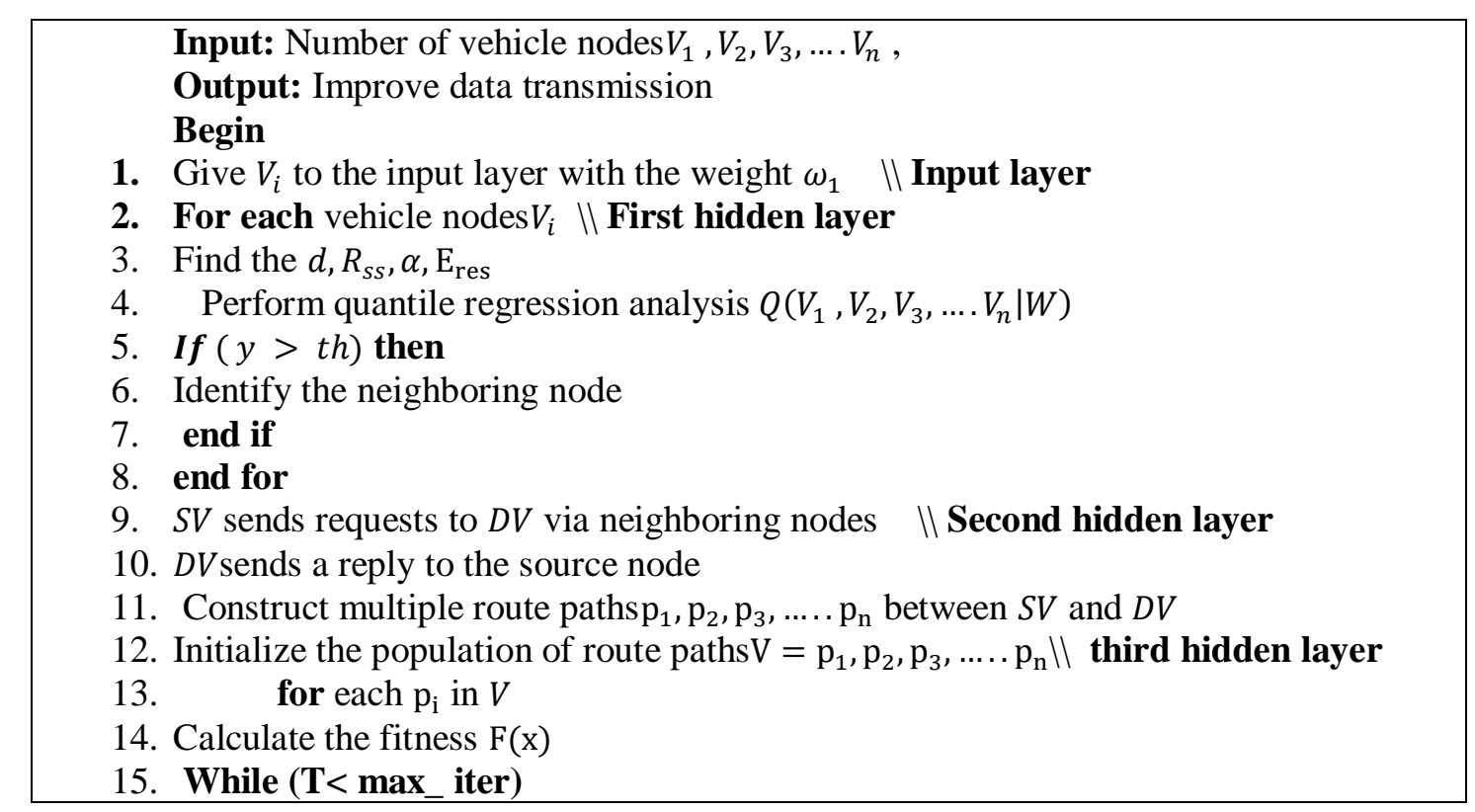




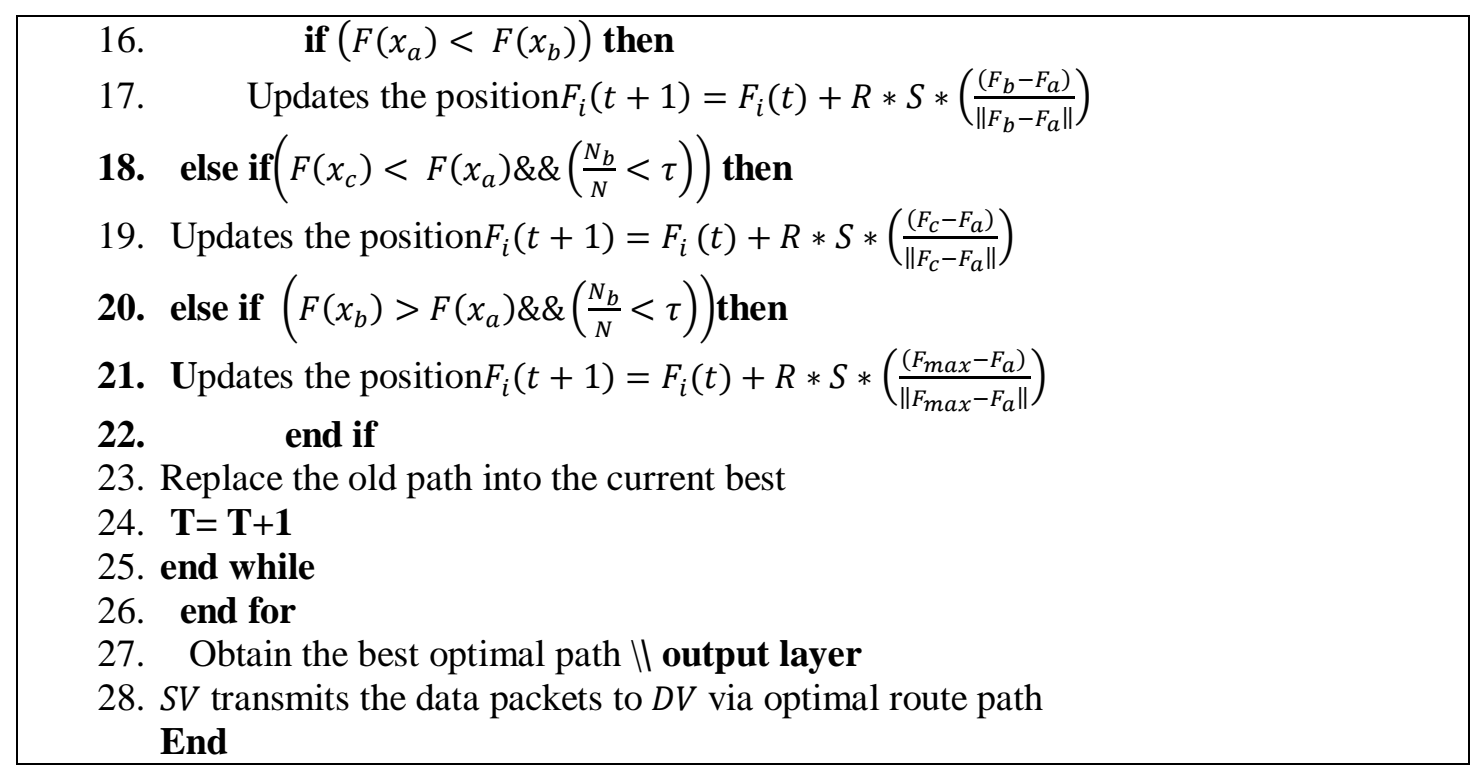

Algorithm 1. Quantile Regressive Fish Swarm Optimized Deep Convolutional Neural Learning

The above-said algorithm clearly describes that the proposed technique effectively finds the route path for reliable data transmission in IoV. In the future, the bandwidth of the node is considered for neighbouring node identification.

\section{Simulation SettingS}

The simulation of the proposed QRFSODCNL technique and existing routing algorithms namely CIoVS [1], QRA [2], EEMSFV [3], IDCIoV [4], Heterogeneous architecture [24],PRCBGWO technique are implemented using NS2.34 network simulator. For the simulation purposes, 500 vehicle nodes are distributed over a squared area of $(1100 m \times 1100 m)$. Random Waypoint is employed as a mobility model. The simulation time is set as $300 \mathrm{sec}$. The Number of vehicle nodes and the number of data packets is considered in the range of 50 to 500 and 30 to 300 respectively. The Size of data packets is $10 \mathrm{~KB}-100 \mathrm{~KB}$. The node speed ranges from $0-20 \mathrm{~m} / \mathrm{s}$. The DSR routing protocol is used to perform optimal route path selection for data transmission in IoV. The simulation parameters and the values are listed below

Table 1. Simulation parameters

\begin{tabular}{|l|l|}
\hline Simulation Parameters & Values \\
\hline Simulator & NS2.34 \\
\hline Simulation area & $1100 \mathrm{~m} * 1100 \mathrm{~m}$ \\
\hline Number of vehicle nodes & $50,100,150,200,250,300,350,400$ \\
& $, 450,500$ \\
\hline Number of data packets & $30,60,90,120,150,180,210,240$, \\
& 270,300 \\
\hline Size of data packet & $10 \mathrm{~KB}-100 \mathrm{~KB}$ \\
\hline Mobility model & Random Waypoint model \\
\hline Speed of nodes & $0-20 \mathrm{~m} / \mathrm{s}$ \\
\hline Simulation time & $300 \mathrm{sec}$ \\
\hline Routing protocol & DSR \\
\hline Number of runs & 10 \\
\hline
\end{tabular}




\section{Comparative Results Analysis}

The results of QRFSODCNL technique and existing CIoVS [1], QRA [2], EEMSFV [3], IDCIoV [4], Heterogeneous architecture [24], PRCBGWO technique are evaluated with metrics namely packet delivery ratio, packet loss rate, end to end delay and throughput. The various results of proposed and existing are discussed with the help of table format and graphical illustration.

\subsection{Packet delivery ratio}

The packet delivery ratio is referred to as the ratio of several packets received to the total number of packets sent. PDR is expressed as follows,

$$
P D R=\left(\frac{N_{D P R}}{N_{D P S}}\right) * 100
$$

Where $P D R$ indicates packet delivery ratio, $N_{D P S}$ indicates the number of data packets sent, $N_{D P R}$ indicates the number of data packets received. PDR is calculated in percentage (\%).

Table 2 illustrates the results of the Packet delivery ratio with the number of data packets. For conducting a simulation, 30 to 300 data packets are taken as input. The reported results prove that the packet delivery ratio of the QRFSODCNL technique is compared to CIoVS [1], QRA [2], EEMSFV [3], IDCIoV [4], Heterogeneous architecture [24], PRCBGWO technique.

Table 2. Packet delivery ratio

\begin{tabular}{|l|l|l|l|l|c|l|l|}
\hline \multirow{2}{*}{$\begin{array}{l}\text { No. } \\
\text { of data } \\
\text { packets }\end{array}$} & \multicolumn{7}{|c|}{ Packet delivery ratio (\%) } \\
\cline { 2 - 8 } & CIoVS & QRA & EEMSFV & IDCIoV & $\begin{array}{l}\text { Heterogeneous } \\
\text { Architecture }\end{array}$ & $\begin{array}{l}\text { PRCB } \\
\text { GWO }\end{array}$ & $\begin{array}{l}\text { QRFSO } \\
\text { DCNL }\end{array}$ \\
\hline 30 & 70 & 77 & 73 & 80 & 81 & 83 & 87 \\
\hline 60 & 75 & 80 & 78 & 83 & 82 & 87 & 90 \\
\hline 90 & 76 & 81 & 80 & 84 & 83 & 88 & 91 \\
\hline 120 & 80 & 83 & 82 & 86 & 87 & 91 & 92 \\
\hline 150 & 81 & 84 & 83 & 87 & 88 & 92 & 93 \\
\hline 180 & 83 & 86 & 85 & 89 & 90 & 93 & 94 \\
\hline 210 & 84 & 88 & 87 & 90 & 92 & 94 & 95 \\
\hline 240 & 85 & 89 & 88 & 91 & 93 & 95 & 96 \\
\hline 270 & 87 & 90 & 89 & 92 & 93 & 96 & 97 \\
\hline 300 & 88 & 91 & 90 & 93 & 95 & 97 & 98 \\
\hline
\end{tabular}




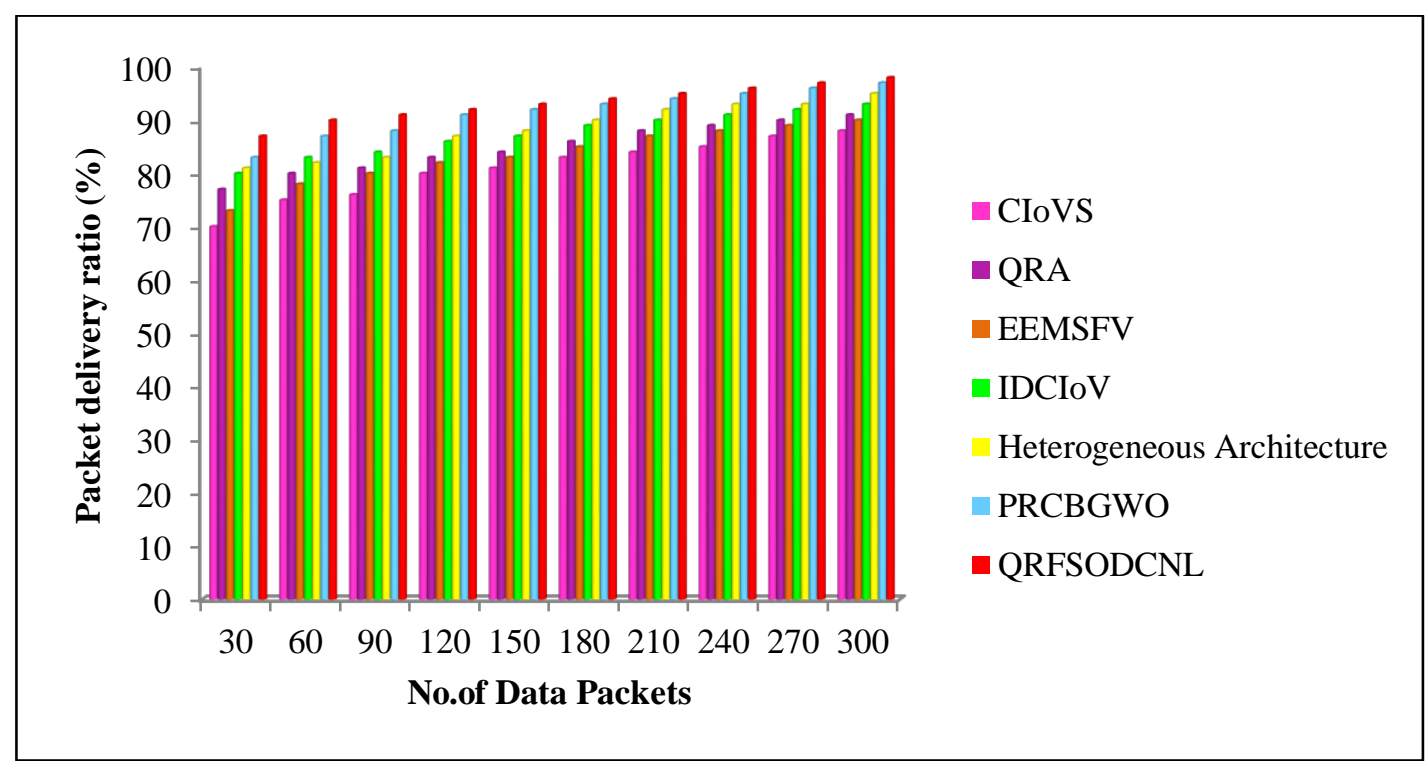

Figure 2. Simulation results of packet delivery ratio

Figure 2 shows the impact of the packet delivery ratio versus the number of data packets sent from the source node. As shown in the above graphical results, the number of data packets taken as input in the horizontal axis, and the results of the packet delivery ratio is obtained at a vertical axis. The graphical results show that the QRFSODCNL technique achieves a higher packet delivery ratio than the other routing methods. This is because the QRFSODCNL technique uses the deep learning concept for neighbour node discovery and the optimal route path identification. The quantile regression function is applied for analyzing the different characteristics and identifying the neighbouring nodes from source to destination. Then the multi-criteria optimization technique is applied for finding the route path and the source node transmitting the data packets to the destination. This, in turn, improves reliable data packet delivery. Ten simulation results of packet delivery ratio are obtained with various input data packets. The average of ten simulation results proves that the packet delivery ratio using QRFSODCNL technique is significantly improved by $16 \%, 10 \%$ and $12 \%$ when compared to CIoVS [1], QRA [2], EEMSFV [3] and 7\%, 2\%, and 6\% as compared to IDCIoV [4], PRCBGWO, Heterogeneous architecture [24].

\subsection{Packet loss rate}

PLR is referred to as the ratio of the number of packets dropped at the destination end to the total number of packets sent. The packet loss rate is measured using the following mathematical equation,

$$
P L R=\left(\frac{N_{D P D}}{N_{D P S}}\right) * 100
$$

Where PLRrepresents the packet loss rate, $N_{D P S}$ denotes the number of data packets sent, $N_{D P D}$ denotes the number of data packets dropped. The packet loss rate is measured in terms of percentage $(\%)$. 


\begin{tabular}{|l|l|l|l|l|c|l|l|}
\hline $\begin{array}{l}\text { No.of } \\
\text { Data } \\
\text { packets }\end{array}$ & CIoVS & $\begin{array}{l}\text { QR } \\
\text { A }\end{array}$ & $\begin{array}{l}\text { EEMSF } \\
\text { V }\end{array}$ & $\begin{array}{l}\text { IDCIo } \\
\text { V }\end{array}$ & $\begin{array}{l}\text { Heterogeneou } \\
\text { s Architecture }\end{array}$ & $\begin{array}{l}\text { PRCB } \\
\text { GWO }\end{array}$ & $\begin{array}{l}\text { QRFSO } \\
\text { DCNL }\end{array}$ \\
\hline 30 & 30 & 23 & 27 & 20 & 19 & 17 & 13 \\
\hline 60 & 25 & 20 & 22 & 17 & 16 & 13 & 10 \\
\hline 90 & 24 & 19 & 20 & 16 & 15 & 12 & 9 \\
\hline 120 & 20 & 17 & 18 & 14 & 13 & 9 & 8 \\
\hline 150 & 19 & 16 & 17 & 13 & 12 & 8 & 7 \\
\hline 180 & 17 & 14 & 15 & 11 & 10 & 7 & 6 \\
\hline 210 & 16 & 12 & 13 & 10 & 9 & 6 & 5 \\
\hline 240 & 15 & 11 & 12 & 9 & 7 & 5 & 4 \\
\hline 270 & 13 & 10 & 11 & 8 & 5 & 4 & 3 \\
\hline 300 & 12 & 9 & 10 & 7 & 4 & 3 & 2 \\
\hline
\end{tabular}

PLR Results of different methods are portrayed in table 3 with the number of the data packet. The various results of the packet loss rate of the different data packets are shown in Figure 3.

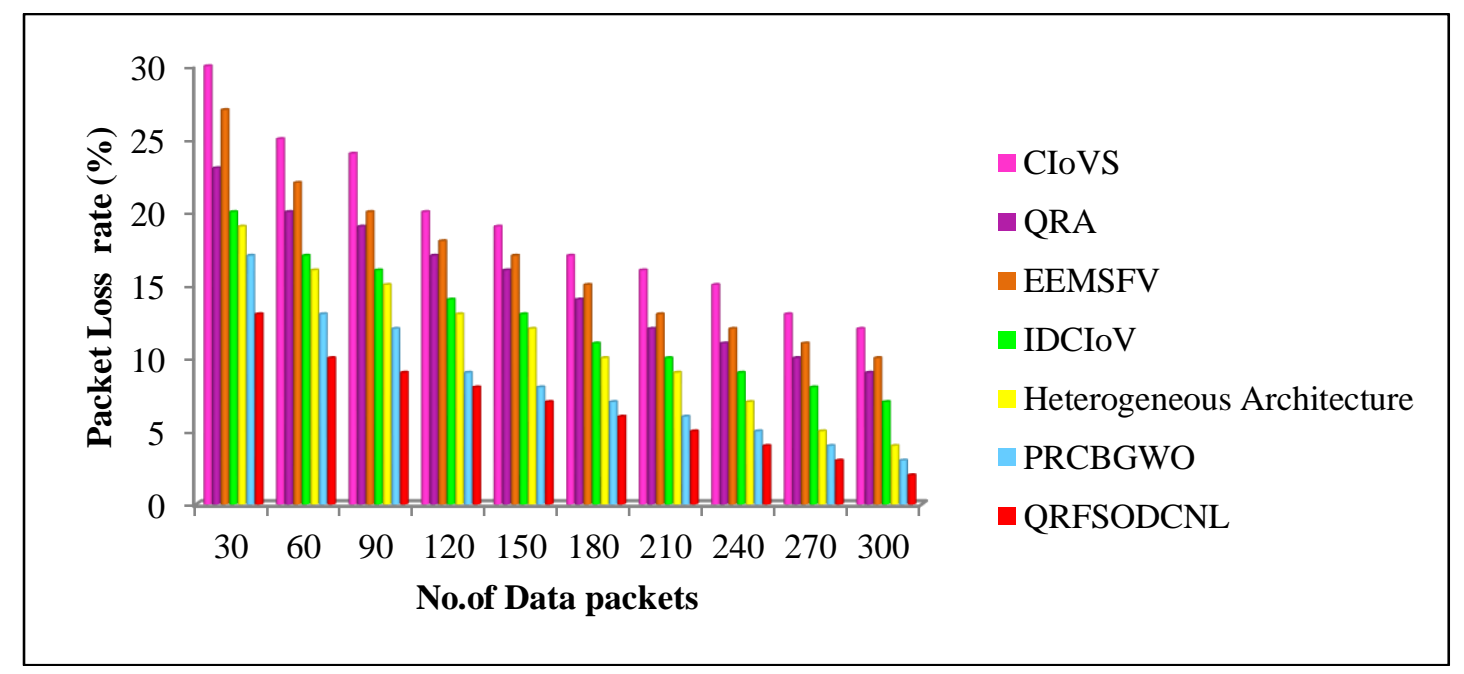

Figure 3. Packet loss rate

Figure 3 depicts the PLR with the number of data packets using different methods. As shown in the above graphical representation, the proposed QRFSODCNL technique minimizes the packet loss rate. This is achieved by considering the available bandwidth, path length, and node buffer capacity. The maximum bandwidth availability and the minimum distance of the route path from source to destination are selected by applying a multi-criteria fish swam optimization. Besides, energy-aware neighbor node selection also improves the data delivery between the vehicles and minimizes the packet drop rate. This is proved by using mathematical calculations. Let us consider 30 data packets being sent from the source node. There are 4 data packets are dropped at the destination end and the packet loss rate is $13 \%$. This shows that the QRFSODCNL technique minimizes the packet loss. The packet loss rate of the proposed QRFSODCNL technique is compared to existing methods. The average of ten results confirms that the packet loss rate of QRFSODCNL technique is reduced by $67 \%, 58 \%, 62 \%, 49 \%, 41 \%$ and $20 \%$ as compared to CIoVS [1], QRA [2], EEMSFV [3], IDCIoV[4], Heterogeneous architecture [24], and PRCBGWO respectively. 


\subsection{End to end delay}

End to end delay is referred to the time difference between the data packet arrival and data packet transmission. The end to end delay is computed using the following mathematical formula,

$$
E E D=\left(T_{D p}(A)-T_{D p}(S)\right)(20)
$$

WhereEED denotes an end to end delay, $T_{D p}(A)$ represents the data packet arrival time, $T_{D p}(S)$ denotes a data packet sending time. EED is measured in milliseconds (ms). The simulation results of end to end delay of different methods with the number of data packets. By applying the 30 data packets, the proposed QRFSODCNL technique obtains $16 \mathrm{~ms}$ of end to end delay.

Table 4. End to end delay

\begin{tabular}{|c|c|c|c|c|c|c|c|}
\hline \multirow{2}{*}{$\begin{array}{c}\text { No. of } \\
\text { data } \\
\text { packets }\end{array}$} & \multicolumn{9}{|c|}{$\begin{array}{c}\text { End to end delay (ms) } \\
\text { S }\end{array}$} & $\begin{array}{c}\text { QR } \\
\text { A }\end{array}$ & EEMSFV & $\begin{array}{c}\text { IDCIo } \\
\text { V }\end{array}$ & $\begin{array}{c}\text { Heterogeneous } \\
\text { Architecture }\end{array}$ & $\begin{array}{c}\text { PRCB } \\
\text { GWO }\end{array}$ & $\begin{array}{c}\text { QRFSODC } \\
\text { NL }\end{array}$ \\
\hline 30 & 26 & 22 & 24 & 20 & 19 & 18 & 16 \\
\hline 60 & 35 & 30 & 32 & 27 & 26 & 25 & 23 \\
\hline 90 & 36 & 32 & 33 & 30 & 29 & 28 & 25 \\
\hline 120 & 40 & 37 & 38 & 35 & 34 & 32 & 29 \\
\hline 150 & 43 & 40 & 41 & 38 & 34 & 35 & 33 \\
\hline 180 & 46 & 42 & 43 & 40 & 39 & 37 & 35 \\
\hline 210 & 50 & 45 & 47 & 42 & 41 & 40 & 38 \\
\hline 240 & 52 & 48 & 49 & 46 & 45 & 44 & 42 \\
\hline 270 & 55 & 52 & 53 & 50 & 49 & 47 & 45 \\
\hline 300 & 57 & 54 & 55 & 52 & 51 & 50 & 48 \\
\hline
\end{tabular}

The other methods CIoVS [1], QRA [2], EEMSFV [3], IDCIoV[4], PRCBGWO, Heterogeneous architecture [24] obtains, $26 \mathrm{~ms}, 22 \mathrm{~ms}, 24 \mathrm{~ms}, 20 \mathrm{~ms}$ and $18 \mathrm{~ms}, 19 \mathrm{~ms}$ of delay. The mathematical results show that the end to end delay is found to be minimized using the QRFSODCNL technique than the other methods. The end to end delay of the different methods is shown in Figure 4.

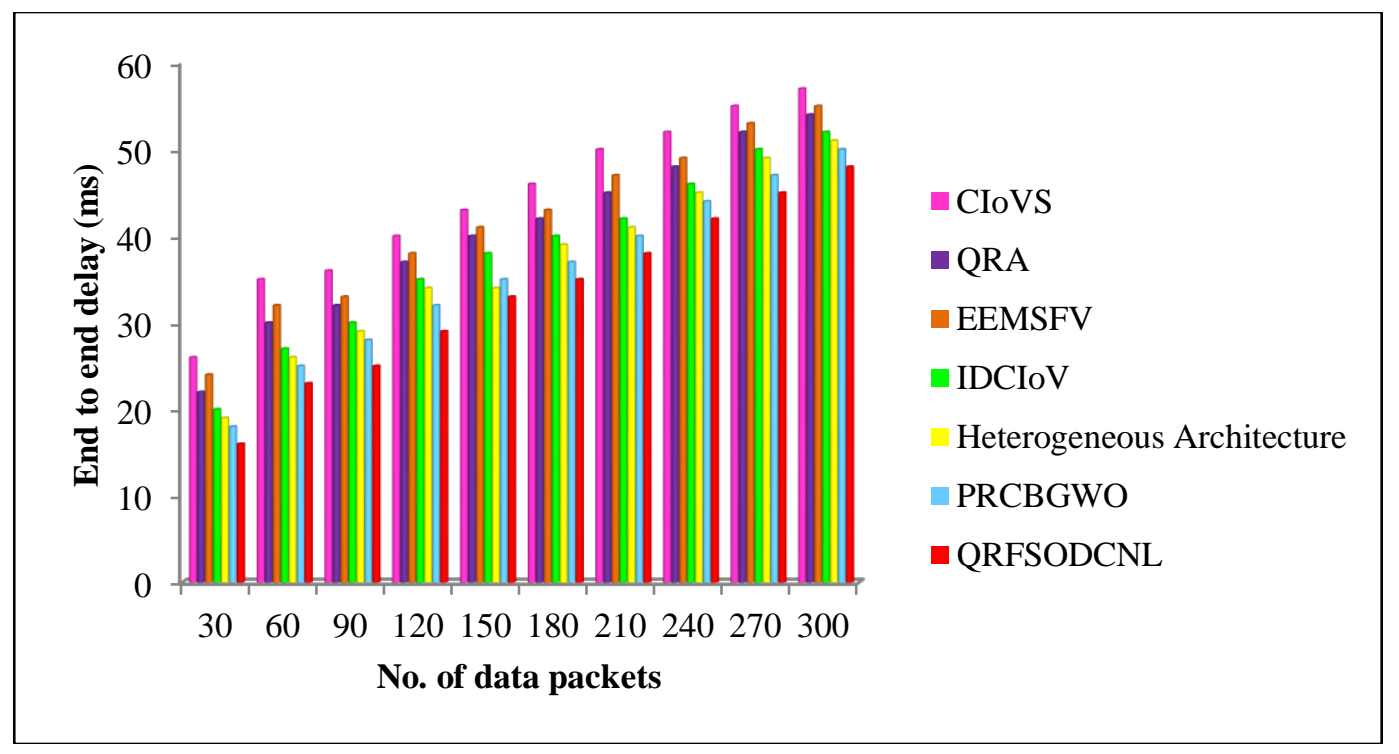

Figure 4. End to end delay 
Figure 4 depicts the number of data packets for different methods. As depicted in the above graphical results, the proposed QRFSODCNL technique provides a minimum end to end delay as compared to existing methods. This is because of applying the quantile regression by analyzing the vehicle node characteristics. The energy-efficient neighbouring node is selected for data transmission. The optimization technique discovers the route path with less distance and hops counts between the source and destination. The source node transmits the data packet to the destination via the minimal number of hop counts and greater bandwidth availability resulting in minimizing the delay. The comparison of ten various results shows that the end to end delay of QRFSODCNL technique is comparatively minimized by $26 \%, 18 \%, 21 \%, 13 \%$ and $10 \%, 7 \%$ as compared to CIoVS [1], QRA [2], EEMSFV [3], IDCIoV[4], Heterogeneous architecture [24],PRCBGWO.

\subsection{Throughput}

Throughput is defined as an amount of data received at the destination within a given period of time. The throughput is calculated as follows,

$$
\text { Throughput }=\frac{\text { AmountofDPR (bits) }}{\mathrm{T}(\mathrm{sec})}(21)
$$

Where $D P R$ represents a data packet received, $T$ denotes a time in seconds (sec). The throughput is measured in the unit of bits per second (bps).

Table 5. Throughput

\begin{tabular}{|l|c|c|c|c|c|c|c|}
\hline $\begin{array}{l}\text { Size of } \\
\text { data } \\
\text { packets } \\
\text { (KB) }\end{array}$ & \multicolumn{7}{|c|}{ Throughput(bps) } \\
\cline { 2 - 8 } & CIoVS & QRA & EEMSFV & IDCIoV & $\begin{array}{l}\text { Heterogeneous } \\
\text { Architecture }\end{array}$ & PRCBGWO & $\begin{array}{l}\text { QRFSO } \\
\text { DCNL }\end{array}$ \\
\hline 30 & 105 & 128 & 120 & 135 & 136 & 142 & 170 \\
\hline 60 & 130 & 180 & 165 & 220 & 230 & 250 & 280 \\
\hline 90 & 195 & 280 & 240 & 310 & 320 & 342 & 370 \\
\hline 120 & 310 & 405 & 360 & 430 & 440 & 463 & 510 \\
\hline 150 & 410 & 480 & 450 & 510 & 520 & 543 & 580 \\
\hline 180 & 500 & 580 & 550 & 620 & 630 & 675 & 710 \\
\hline 210 & 580 & 650 & 620 & 700 & 720 & 741 & 812 \\
\hline 240 & 620 & 720 & 700 & 760 & 890 & 812 & 920 \\
\hline 270 & 730 & 810 & 780 & 890 & 900 & 974 & 1010 \\
\hline 300 & 840 & 980 & 890 & 1056 & 1070 & 1120 & 1150 \\
\hline
\end{tabular}




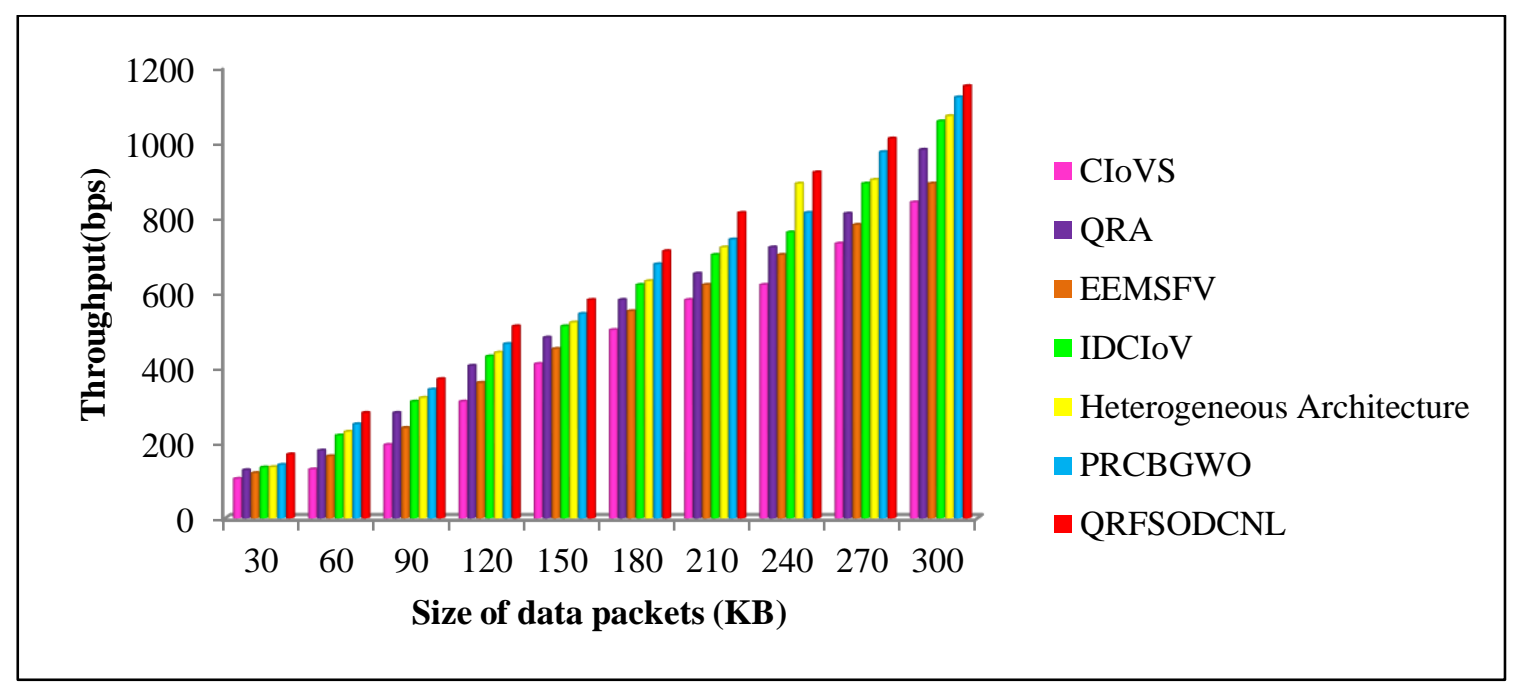

Figure 5. Throughput

Table 5 and figure 5 denotes simulation results of throughput using different methods with various sizes of the data packet. The graphical results of the throughput are found to be increased using the QRFSODCNL technique. The multi-criteria optimization technique discovers the best available route path among source vehicle and destination vehicle via neighbouring nodes. While routing the data packets from the source node, an efficient neighbouring node is selected based on the quantile regression function with various vehicle node characteristics. The selected neighbouring node efficiently transfers the data packets from one to another. This, in turn, improves the data packet transmission. The reported results proves that the throughput is significantly improved by 58\%, 28\%, 39\%, 18\% and 14\%, 9\% when compared to CIoVS [1], QRA [2], EEMSFV [3], IDCIoV [4], Heterogeneous architecture [24], PRCBGWO respectively.

\section{Conclusion}

An efficient deep learning technique QRFSODCNL is introduced to achieve reliable data transmission in IoV and minimal delay as well as packet loss. The number of vehicle nodes is given to the input layer of deep neural learning. The deep convolution neural learning includes one input layer, three hidden layers, and one output layer. At first, the number of vehicles is given as input to the input layer. Then, the inputs are transferred into the first hidden layer. After that, the inputs are learned in hidden layers using the regression analysis with multi-criteria optimization. The node characteristics are analyzed and identify the location of the neighbouring node. Followed by, multiple paths are identified in the second hidden layer from source to destination. Finally, the multi-criteria fish swarm optimization is applied in the third hidden layer to determine the best path for delivering the data packets with minimum delay. The simulation is performed with metrics namely packet delivery ratio, packet loss rate, end-to-end delay, and throughput. The result analysis clearly shows that the QRFSODCNL technique improves reliable data transmission with higher packet delivery ratio and throughput as well as minimizes the packet loss, end to end delay when compared to the state-of-the-art methods. In the future, if any link failure occurred during the data transmission, an alternate route path is considered for data transmission to avoid the packet loss rate.

\section{CONFLICTS OF INTEREST}

The authors declare no conflict of interest. 
International Journal of Computer Networks \& Communications (IJCNC) Vol.13, No.2, March 2021

\section{REFERENCES}

[1] Tie Qiu,XizeLiu,KeqiuLi, QingHu,Arun Kumar Sangaiah, Ning Chen, Community-Aware Data Propagation with Small World Feature for the Internet of Vehicles, IEEE Communications Magazine, 56, 1, 2018, P 86 - 91

[2] KayhanZrarGhafoor, LingheKong,Danda B. Rawat,EghbalHosseini, Ali SafaaSadiq, Quality of Service Aware Routing Protocol in Software-Defined Internet of Vehicles", IEEE Internet of Things Journal, 6, 2, 2019, p $2817-2828$

[3] Ahmed JawadKadhim and Seyed Amin Hosseini Seno, "Energy-Efficient Multicast Routing Protocol based on SDN and Fog computing for Vehicular Networks", Ad Hoc Networks, Elsevier, 84, 2019, p 68-81

[4] S. Suguna Devi and Dr.A.Bhuvaneswari, Identical Destination Based Community on Internet of Vehicles (IDCIoV) for Optimal Path Identification", International Conference on Sustainable Communication Networks and Application, 2019, p 368-378

[5] XiyingFan,ChuanheHuang,Junyu Zhu, and Bin Fu, "Replication-Based Data Dissemination in Connected Internet of Vehicles", Wireless Communications and Mobile Computing, Hindawi, 2019, April 2019, p 1-16

[6] FarhanAadil, WaleedAhsan, Zahoor Ur Rehman, Peer Azmat Shah, Seungmin Rho, IrfanMehmood, "Clustering algorithm for internet of vehicles (IoV) based on dragonfly optimizer (CAVDO)", The Journal of Supercomputing, Springer, 74, 9, 2018, p 4542-4567

[7] Muhammad Fahada, FarhanAadil, Zahoor-ur- Rehman, SalabatKhana, Peer AzmatShah,Khan Muhammad Jaime Lloret, Haoxiang Wang, Jong Weon Lee, IrfanMehmoode, "Grey wolf optimization based clustering algorithm for vehicular ad-hoc networks", Computers \& Electrical Engineering, Elsevier, Volume 70, 2018, p 853-87

[8] YassineHernafi, Mohamed Ben Ahmed, Mohammed Bouhorma, "ACO and PSO Algorithms for Developing a New Communication Model for VANET Applications in Smart Cities", Wireless Personal Communications, Springer, 96, 2, 2017, p2039-2075

[9] ShirinRahnamaeiYahiabadi, BehrangBarekatain\&KaamranRaahemifar, "TIHOO: An Enhanced Hybrid Routing Protocol in Vehicular Ad-hoc Networks", EURASIP Journal on Wireless Communications and Networking, Springer, 2019, p 1-19

[10] Cheng Wang, Luomeng Zhang, Zhong Li, Changjun Jiang, "SDCoR: Software Defined Cognitive Routing for Internet of Vehicles", IEEE Internet of Things Journal, 5, 5, 2018, p 3513 - 3520

[11] DaxinTian, KunxianZheng, Jianshan Zhou, XutingDuan, Yunpeng Wang, Zhengguo Sheng, and Qiang Ni, "A Microbial Inspired Routing Protocol for VANETs", IEEE Internet of Things Journal, 5, 4, 2018, p $2293-2303$

[12] XiuZhanga, XinZhanga, Cheng Gu, "A micro-artificial bee colony based multicast routing in vehicular ad hoc networks", Ad Hoc Networks, Elsevier, 58, 2017, p 213-221

[13] Chen Chen, Tingting Xiao, Mengyuan Zhang, Qingqi Pei, "PTCCR: A Path Transmission CostsBased Multi-Lane Connectivity Routing Protocol for Urban Internet of Vehicles", IEEE Access, 7, 2019, p 141838 - 141849

[14] Nori M. Al-Kharasani, Zuriati Ahmad Zulkarnain, ShamalaSubramaniam and ZurinaMohdHanapi, “An Efficient Framework Model for Optimizing Routing Performance in VANETs", Sensors, 18, 2, 2018, p 1-21

[15] Osama Rehman, and Mohamed Ould-Khaoua, "A hybrid relay node selection scheme for message dissemination in VANETs", Future Generation Computer Systems, Elsevier, 93, 2019, p 1-17

[16] Muhammad Ali, Asad W Malik, Anis U Rahman, Sohaillqbal, and Mian M Hamayun, "Positionbased emergency message dissemination for Internet of vehicles", International Journal of Distributed Sensor Networks, 15, 7, 2019, p 1-12

[17] Wanting Zhu, DeyunGao, ChuanHengFoh,HongkeZhang,Han-Chieh Chao, "Reliable emergency message dissemination protocol for urban internet of vehicles", IET Communications 11, 8, 2017, p $1275-1281$

[18] Celimuge Wu, Tsutomu Yoshinaga, YushengJi, Yan Zhang, "Computational Intelligence Inspired Data Delivery for Vehicle-to-Roadside Communications", IEEE Transactions on Vehicular Technology, 67, 12, 2018, p $12038-12048$ 
International Journal of Computer Networks \& Communications (IJCNC) Vol.13, No.2, March 2021

[19] A. M. Oranj, R. M. Alguliev, FarhadYusifov, ShahramJamali, "Routing Algorithm for Vehicular Ad Hoc Network Based on Dynamic Ant Colony Optimization”, International Journal of Electronics and Electrical Engineering, 4, 1, 2016, p 79-83

[20] Hafiz HusnainRazaSherazi,ZuhaibAshfaq Khan, RaziIqbal, ShahzadRizwan, Muhammad Ali Imran, and Khalid Awan, "A Heterogeneous IoV Architecture for Data Forwarding in Vehicle to Infrastructure Communication", Mobile Information Systems, Hindawi, February 2019, p 1-12

[21] Wanting Zhu, DeyunGao, Weicheng Zhao, Hongke Zhang \&Hua-Pei Chiang, "SDN-enabled hybrid emergency message transmission architecture in internet-of-vehicles", Enterprise Information Systems, 12, 4, 2018, p 1-20

[22] Muhammad Tahir Abbas, Afaq Muhammad, Wang-Cheol Song, "SD-IoV: SDN enabled routing for internet of vehicles in road-aware approach",Journal of Ambient Intelligence and Humanized Computing, 11, 2020, p 1265-1280.

[23] CHUNG-MING HUANG, TZU-HUA LIN , AND KUAN-CHENG TSENG, "Data Dissemination of Application Service by Using Member-Centric Routing Protocol in a Platoon of Internet of Vehicle (IoV)", IEEE Access, 7, 2019, p 127713 - 127727.

[24] Hafiz HusnainRazaSherazi , ZuhaibAshfaq Khan, RaziIqbal, ShahzadRizwan, Muhammad Ali Imran and Khalid Awan, "A Heterogeneous IoV Architecture for Data Forwarding in Vehicle to Infrastructure Communication”, Hindawi, Mobile Information Systems,2019,2019, p 1-12.

\section{AUTHORS}

MS. S. Suguna Devi pursued a Master of Computer Applications from Bharathidasan University in the year 1999. She is currently pursuing a Ph.D. and working as an Assistant Professor in the Department of Computer Science, Cauvery College for Women Trichy, Tamil Nadu, India. She has15 years and teaching experience. She is a life member of ISSE. Her main research work focuses on Internet of Vehicles.

Dr. A. Bhuvaneswari completed her Master's Degree in Computer Science and Master of Philosophy in Computer Science in the year 2002 and 2005 respectively. She has also completed her Doctorate in Computer Science in the year 2015. She has around 18 years of Academic experience and eleven years of Research experience in the field of Computer Science. Currently, she is working at Cauvery College for Women (under the affiliation of Bharathidasan University), Trichy, Tamil Nadu, India. She has published several papers in International

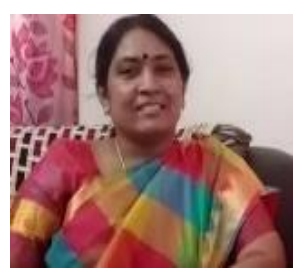
Journals and Conferences related to Computer Science. Her area of interest is Mobile Communication and the Internet of Things. 